REVISTAS DE LA FAHCE

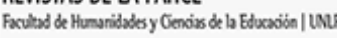

? ?
Educación Física y Ciencia

ISSN: 1514-0105

ISSN: 2314-2561

revistaefyc@fahce.unlp.edu.ar

Universidad Nacional de La Plata

Argentina

\title{
Prácticas inclusivas de estudiantes en formación docente
}

\author{
Márquez Cabellos, Norma Guadalupe; Andrade Sánchez, Adriana Isabel \\ Prácticas inclusivas de estudiantes en formación docente \\ Educación Física y Ciencia, vol. 22, núm. 1, 2020 \\ Universidad Nacional de La Plata, Argentina \\ Disponible en: http://www.redalyc.org/articulo.oa?id=439963095001 \\ DOI: https://doi.org/10.24215/23142561e109
}

Esta obra está bajo una Licencia Creative Commons Atribución-NoComercial-Compartirlgual 4.0 Internacional. 


\section{Prácticas inclusivas de estudiantes en formación docente}

Inclusive practices of students in teacher training

Norma Guadalupe Márquez Cabellos

Universidad de Colima, México

DOI: https://doi.org/10.24215/23142561e109

norma_marquez@ucol.mx

Redalyc: http://www.redalyc.org/articulo.oa?

Adriana Isabel Andrade Sánchez

Universidad de Colima, México

id $=439963095001$

Recepción: 15 Octubre 2019

Aprobación: 11 Febrero 2020

\section{RESUMEN:}

Ofrecer una educación de calidad, igualdad, equidad y pertinencia no es tarea fácil para los profesores de estudiantes en formación docente; porque implica desafíos en el cómo enseñar, en el qué y cómo evaluar desde una visión inclusiva. Se presenta una investigación cuantitativa sobre las prácticas inclusivas en la formación docente de estudiantes inscritos en las tres licenciaturas que oferta la Facultad de Ciencias de la Educación de la Universidad de Colima: educación física y deporte, enseñanza de las matemáticas y educación especial. Participaron 269 estudiantes cumplimentando la Guía de evaluación de las prácticas inclusivas en el aula -GEPIA- (García, Romero y Escalante, 2011). Se identificó que existen diferencias altamente significativas de la perspectiva que tienen los estudiantes de las tres licenciaturas en relación a las buenas prácticas inclusivas; específicamente en las categorías que refiere la GEPIA. Se concluye en la importancia de ofrecer herramientas teóricas metodológicas a estudiantes en formación docente para atender las particularidades y características de los alumnos de la escuela regular; es decir, centrar la atención de una educación para todos.

Palabras Clave: Formación docente, Educación inclusiva, Prácticas inclusivas.

\section{Abstract:}

Offering quality education, equality, equity and relevance is not an easy task for professors of students in teacher education; because it implies challenges in how to teach, in what and how to evaluate from an inclusive vision. A quantitative investigation is presented on the inclusive practices in the training of students enrolled in the three degrees offered by the Faculty of Education Sciences of the University of Colima: Sport and Physical Education, Math Teaching and Special Education. 269 students participated completing the Guidelines for the Evaluation of Inclusive Practices in the Classroom -GEPIA- (García, Romero y Escalante, 2011). It was identified that there are highly significant differences in the perspective of the students of the three degrees in relation to good inclusive practices. We conclude that it is important to offer methodological theoretical tools to students in teacher training to attend the particularities and characteristics of the students of regular school; that is, to focus the attention of an education for all. KEYWORDS: Teacher training, Inclusive education, Inclusive practices.

\section{INTRODUCCIÓN}

La educación superior que brindan las instituciones públicas o privadas están comprometidas a identificar las áreas de oportunidad del presente, proyectar su futuro, a repensarse y a reorganizarse de forma creativa, estratégica e innovadora para atender a las exigencias que demanda una sociedad en rápida transformación. Las acciones de la educación superior antes señaladas nos lleva a pensar en la educación inclusiva entendida como

un proceso sin fin que tiene que ver con la participación de las personas, la creación de sistemas de participación y sus ajustes, y la promoción de valores inclusivos. Se trata de aumentar la participación de todos en las culturas, las comunidades y el curriculum y la reducción de todas las formas de exclusión y discriminación (Booth \& Ainscow, 2015, p. 24). 
En efecto, se considera como un proceso sistemático que implica la toma de decisiones para la mejora educativa en la transformación de prácticas pedagógicas, el logro de desempeños de estudiantes, el diseño de un curriculum flexible e innovador que responda a los cambios vertiginosos de la sociedad. Jiménez (2014) refiere que "la inclusión se mueve como dos placas tectónicas que en su encuentro cimbran la tierra provocando desastres naturales" (p. 69). Ésta es la realidad de muchas instituciones educativas de nuestro México. Por ello, para lograr la inclusión se requiere una sociedad con pensamiento incluyente y no segregacionista, donde se promuevan condiciones que permitan que cada persona se sienta valorado por igual. Por ello, partimos de la premisa que las prácticas inclusivas se deben gestar en las aulas de los formadores en docencia, en un curriculum flexible con diseño universal de una educación para todos, así como en la política educativa para marcar las pautas de las acciones a emprender en las aulas, donde el docente identifique las áreas de oportunidad y particularidades de todos sus alumnos; lo que le permitirá una transformación pedagógica centrada en la diversidad escolar.

El movimiento de la educación inclusiva trabaja desde las potencialidades del estudiante con discapacidad, trastorno, aptitudes sobresalientes y; todo aquel o aquella que presente una condición social, cultural, física, cognitiva que limite su aprendizaje y su participación. Bajo este precepto, todos somos diferentes; por tanto se busca responder a las necesidades de todos y todas defendiendo el derecho de las personas a acceder a una educación integral que permita desarrollar al máximo las capacidades y potencialidades, eliminando cualquier barrera que impida el proceso de aprendizaje y participación (Booth \& Ainscow, 2015). Por ende, la educación inclusiva requiere no sólo estar presente en cuaderno y/o manual de operación institucional; sino participar de manera efectiva y activa cambiando mentalidades en la práctica pedagógica; en el curriculum, en la estructura organizativa y administrativa, así como fomentar el uso de estrategias metodológicas cooperativas; especialmente transformadoras del contexto educativo.

Si bien es cierto, durante estos últimos años hemos presenciado cambios en el curriculum de las instituciones formadoras de docentes, tal es el caso de las Escuelas Normales o en las Universidades públicas que ofertan licenciaturas afines a la educación. Se identifican documentos curriculares centrados en atender a la diversidad, sin embargo, las prácticas pedagógicas de los estudiantes en formación no suelen estar libre de dificultades y barreras que limitan el ejercicio pedagógico en las aulas regulares. Muchos estudiantes en formación docente no cuentan con las habilidades y/o competencias para atender la diversidad escolar, porque carecen de estrategias teóricas metodológicas centradas en el ejercicio de buenas prácticas inclusivas que responda a la implementación de ajustes razonables para quienes lo requieran. Atender a la diversidad desde el enfoque de la educación inclusiva implica trabajo coordinado y colaborativo con todos los actores de la educación; porque no basta con permitir el acceso de todos los alumnos sin introducir planteamientos pedagógicos e institucionales que garanticen la igualdad y equidad educativa. Por tanto, sin estas premisas se corre el riesgo de que se produzca la exclusión dentro de la inclusión; por ello la importancia que las escuelas formadoras en docencia desarrollen competencias genéricas y específicas en sus estudiantes para atender a la diversidad. Un tema álgido y discutido en la educación -en cualquier nivel educativo- ha sido las prácticas inclusivas; por ello la mirada en el presente documento, está centrada en detectar las prácticas inclusivas en la preparación académica de futuros docentes.

Los estudiantes que se forman como docentes enfrentan un sin fin de desafíos, y uno de ellos es la educación inclusiva porque implica reflexionar sobre las competencias que todo educador debe tener para atender a la población en condición de vulnerabilidad; es decir, la intervención de prácticas inclusivas. Pero epor qué hablar de prácticas inclusivas como un desafío? Sin lugar a duda, porque implica que los futuros docentes construyan o re-construyan dinámicas del proceso enseñanza-aprendizaje a partir del principio de equidad, "no ofrecer lo mismo a todos los alumnos sino ofrecerles lo que necesitan, de una manera diferenciada y en equilibrio para que cada uno de ellos alcance los niveles educativos que correspondan a sus potenciales de aprendizaje (Secretaría de Educación Pública, 2006, p. 22). Desde nuestro punto de vista, la educación inclusiva permite que toda la comunidad escolar se sienta cómodo ante la diversidad, identificándola como 
un desafío y una oportunidad para enriquecer las formas de enseñar y aprender. Así pues, la inclusión es un proceso y tiene que ser vista como una búsqueda constante de mejores maneras de responder a la diversidad de los alumnos, aprendiendo a tratar las diferencias y adaptándolas de manera que se cubran las necesidades de todos que lo requieran. En relación a la práctica docente Lopes (2015) expresa la necesidad de "atender la formación inicial del profesorado al ser una de las variables determinantes en la calidad de la educación inclusiva" (p. 153). Por su parte Orjuela (2009) plantea que hoy día la educación inclusiva supone diversas transformaciones no sólo en el aula, sino en la formación inicial de quienes serán profesores, porque eso contribuirá a una educación de calidad para todos, en donde la formación pedagógica se convierta en un factor fundamental para ese propósito. Así pues, las prácticas inclusivas comprenden todos los elementos implicados en el proceso enseñanza-aprendizaje, y las diversas cuestiones en las que éste ocurre y mediante las que interactúa la población infantil.

Partimos de la premisa que las instituciones de educación superior son factores claves en la sociedad del conocimiento, por ello deben dotarse de fundamentos teóricos y metodológicos para incrementar permanentemente su capacidad de adaptación en un mundo dinámico, y así responder con urgencia a la velocidad de cambios y enfoques educativos, sociales, políticos, económicos y culturales (Márquez, Vargas y Ramos, 2016). Actualmente, se puede identificar en los diferentes foros mundiales la discusión por el futuro de la educación superior a partir de las condiciones presentes y perspectivas futuras de una sociedad demandante y diversificada, asociada a un mundo globalizado en permanente cambio, como resultado de los avances científico-tecnológicos que se generan día con día. En las reuniones convocadas por la Organización de las Naciones Unidas para la Educación, la Ciencia y la Cultura-UNESCO- (2004), el Informe de Delors en 1996, la Conferencia Mundial sobre la Educación Superior celebrada en París en 1998 y en julio de 2009, se precisa la necesidad de una transformación universitaria mundial poniendo de manifiesto la preocupación por la calidad, la pertinencia del quehacer de las Instituciones de Educación Superior (IES), la urgencia de mejorar sustancialmente los procesos de gestión y administración, la necesidad de incorporar las nuevas tecnologías de la información y comunicación y la conveniencia de fortalecer la dimensión internacional de la educación superior. Por tanto, el compromiso que tienen estas instituciones es formar profesionales que cuenten con las competencias básicas para desarrollarse en un mundo cada vez más competitivo, demandante y cambiante, disponiendo de una amplia gama de herramientas, habilidades y capacidades que les permita a los jóvenes profesionistas mantenerse a la vanguardia y realizar proyectos y propuestas innovadoras para triunfar en un mundo de constante cambios. Bajo esta premisa, las instituciones de educación superior deben organizar su tarea cotidiana; desde el diseño del curriculum inclusivo hasta los ajustes razonables en el proceso enseñanza aprendizaje; así como la eliminación de barreras arquitectónicas, sociales y actitudinales para que todos los estudiantes tengan la posibilidad de desarrollar al máximo sus potencialidades.

Desde esta perspectiva, la Universidad de Colima se ha caracterizado a lo largo de su historia en asumir los retos que le plantea una sociedad dinámica y cambiante, tal como se expresa en su visión: "La Universidad de Colima en el 2030 es una institución reconocida mundialmente como una de las mejores universidades del país por su calidad y pertinencia, que asume su responsabilidad social contribuyendo al desarrollo equitativo, democrático y sustentable de la entidad, la nación y el mundo" (Universidad de Colima, 2010, p. 37); desde esta postura se tiene una perspectiva humanista, con una formación innovadora centrada en el aprendizaje y con amplios esquemas de flexibilidad que permiten diversificar las opciones de formación, el desarrollo de competencias necesarias para la vida y el trabajo, logrando eficientar el uso de los recursos, ligados siempre a un esquema moderno de gestión educativa.

Bajo esta perspectiva humanista y flexible ¿qué implica para la Universidad de Colima habilitar académicamente a sus estudiantes en formación docente en la construcción de buenas prácticas inclusivas? implica una infinidad de acciones que impacten en el ejercicio de su profesión, donde se generen cambios en la actitud docente, implementen programas de intervención para los estudiantes con discapacidad, trastorno, aptitudes sobresalientes o en situación de vulnerabilidad; coadyuvando de esta manera a satisfacer 
las necesidades básicas de aprendizaje. Es por ello, que la Máxima Casa de Estudios del estado de Colima, México consiente de la responsabilidad social que tiene ha propuesto que los planes y programas de estudio de los futuros docentes se construya desde un enfoque incluyente, donde los egresados cuenten con las competencias genéricas y especificas para atender a la diversidad de población en las aulas regulares.

Con sustento en lo anterior, las Facultades y Escuelas de la Universidad de Colima puntualizan la inclusión educativa como proceso vinculado con la planeación, desarrollo del curriculum y el aprendizaje; centrando su atención al logro de los aprendizajes de sus estudiantes. Ante este postulado, vale la pena reflexionar ¿qué prácticas inclusivas implementan los estudiantes en formación docente? Sin duda, la inclusión es una tarea compartida que nunca termina, su razón de ser es erradicar la discriminación, marginación y segregación para lograr la promoción del aprendizaje y la participación de todos los niños, jóvenes y adultos en cualquier contexto en el que se desenvuelvan. Las prácticas inclusivas deben entenderse en función del contexto educativo donde se pretendan ejecutar, según lo comenta la Organización de Estados Iberoamericanos. García, Romero, Rubio, Flores y Martínez (2015) refieren que las prácticas inclusivas son actividades situadas, viables y en contextos bien definidos, por lo que una buena práctica puede no serlo en otra escuela o contexto diferente al analizado. En este sentido el presente estudio tiene como objetivo detectar las prácticas inclusivas de los estudiantes en formación docente de las licenciaturas en educación física y deporte, enseñanza de las matemáticas y educación especial; al ser las tres carreras que oferta la Universidad de Colima cuyo perfil profesional está centrado en la adquisición de competencias profesionales y específicas centradas en la docencia en niveles de educación básica y media superior.

\section{Metodología}

El presente estudio es de tipo cuantitativo. El objetivo de la investigación estuvo centrado en identificar las prácticas inclusivas de los estudiantes en formación docentes de la Facultad de Ciencias de la Educación de la Universidad de Colima, México. Para la recolección de datos se aplicó la Guía de evaluación de prácticas inclusivas en el aula -GEPIA- (García, Romero y Escalante, 2011). Esta Guía es una adaptación del índice de inclusión de Booth y Ainscow (2002). Se conforma de seis categorías: condiciones físicas del aula, planeación y uso del tiempo, metodología, evaluación, relación entre el maestro y los alumnos, los alumnos y los maestros entre sí y reflexión y sensibilización. Consta de 58 reactivos tipo Likert que van desde totalmente cierto (con valor de cuatro puntos), parcialmente cierto (tres puntos) parcialmente falso (dos puntos) y totalmente falso (con el valor de uno).

El estudio se realizó en las aulas de la Facultad de Ciencias de la Educación de la Universidad de Colima, México participando 269 estudiantes ( 83 hombres y 186 mujeres). El desglose de participantes corresponde a 127 de la licenciaturas de educación especial, 68 de enseñanza de las matemáticas y 74 de educación física y deporte. Los datos fueron analizados empleando el Statistical Package for the Social Sciences (SPSS v.20) para obtener los promedios de las respuestas de las poblaciones y desviaciones estándar. También se aplicó la T de Student y ANOVA para la comparación de medias.

\section{Resultados}

Para el análisis del instrumento aplicado, se implementó la suma por categoría y los promedios de la respuesta de las poblaciones de las tres licenciaturas. Para analizar si existían diferencias estadísticas entre sexos se utilizó la T de Student para la comparación de medias y; ANOVA para las diferencias entre las carreras en formación docente que oferta la Facultad de Ciencias de la Educación de la Universidad de Colima, México.

La tabla 1 muestra los promedios y desviaciones estándar de los 83 hombres encuestados y las 186 mujeres, pertenecientes a las tres licenciaturas y el p-valor resultante. Tras analizar los resultados con un nivel de 
confianza del $95 \%$ se puede observar como las seis categorías de la GEPIA tiene resultados significativamente distintos entre hombres y mujeres ( $\mathrm{p}$-valor $<0.05$ ), por tal motivo es importante resaltar en la misma tabla que las mujeres perciben que las condiciones físicas del aula, la planeación y uso del tiempo, metodología, evaluación, relación entre el maestro y los alumnos así como la reflexión y sensibilización son adecuadas para tener buenas prácticas inclusivas; en cambio hay más hombres que indican que estas afirmaciones son parcialmente ciertas.

Tabla 1. Comparativo entre sexos

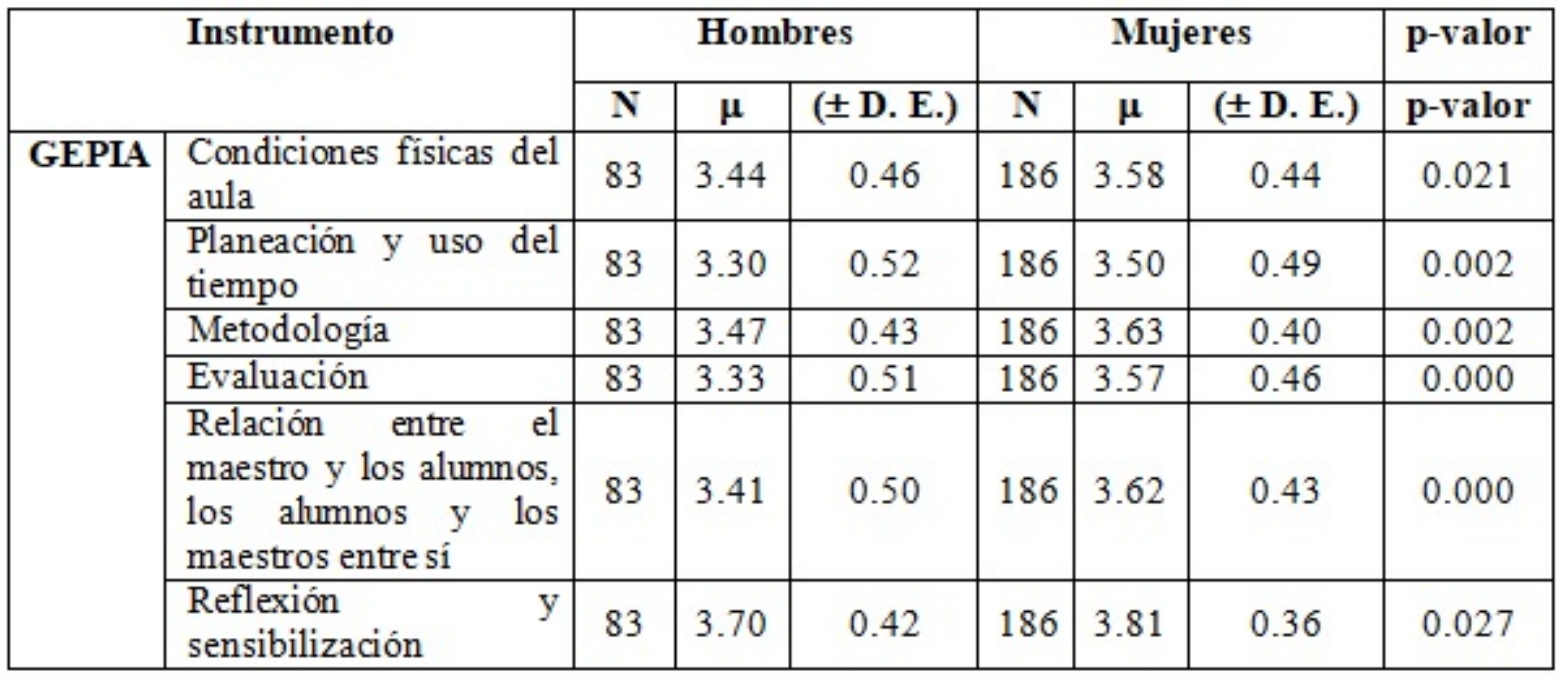

Haciendo un comparativo de las seis dimensiones de la GEPIA entre las tres licenciaturas en formación docente que oferta la Facultad de Ciencias de la Educación, los resultados del análisis de ANOVA se obtuvo un nivel de confianza del $95 \%$ con valores altamente significativos ( $\mathrm{p}$-valores $<0.010$ ) entre las tres carreras. En la tabla 2 se identifica que los estudiantes de la Licenciatura en educación especial otorgan puntuaciones más elevadas en comparación con el resto de los estudiantes en las seis dimensiones de la GEPIA.

Para conocer exactamente entre qué licenciaturas se encontraban las diferencias estadísticas, se realizó la prueba post hoc de Tukey. A través de sus resultados se puede afirmar que en lo que refiere a las condiciones físicas del aula, metodología y reflexión y sensibilización no hay diferencias significativas ( $\mathrm{p}$-valor $>0.050$ ) entre la Licenciatura en educación física y deporte (LEFyD) y la Licenciatura en enseñanza de las matemáticas (LEM); sin embargo, sí las hay entre estas dos licenciaturas y la Licenciatura en educación especial (LEE) (pvalor $<0.010)$. En cuanto a la planeación y uso del tiempo, evaluación y la relación entre el maestro y los alumnos, los alumnos y los maestros entre sí se encontraron diferencias entre las tres licenciaturas ( $\mathrm{p}$-valor $<$ 0.050). En todos los casos las promedios de puntuación más baja la otorgan los alumnos de la Licenciatura en enseñanza de las matemáticas y las más altas los alumnos de la Licenciatura en educación especial. 
Tabla 2. Perspectiva de prácticas inclusivas según la licenciatura

\begin{tabular}{|c|c|c|c|c|c|c|c|c|c|c|c|}
\hline \multicolumn{2}{|c|}{ Instrumento } & \multicolumn{3}{|c|}{ LEFyD } & \multicolumn{3}{|c|}{ LEM } & \multicolumn{3}{|c|}{ LEE } & \multirow[b]{2}{*}{$\begin{array}{c}\text { p- } \\
\text { valor }\end{array}$} \\
\hline & & $\mathbf{N}$ & $\mu$ & $\begin{array}{l}\text { ( } \pm \text { D. } \\
\text { E. })\end{array}$ & $\mathbf{N}$ & $\mu$ & $\begin{array}{l}\text { ( } \pm \text { D. } \\
\text { E. })\end{array}$ & $\mathbf{N}$ & $\mu$ & $\begin{array}{l}\text { ( } \pm \text { D. } \\
\text { E. })\end{array}$ & \\
\hline \multirow[t]{6}{*}{ GEPIA } & $\begin{array}{l}\text { Condiciones } \\
\text { físicas del aula }\end{array}$ & 74 & 3.47 & 0.45 & 68 & 3.38 & 0.52 & 127 & 3.66 & 0.38 & 0.000 \\
\hline & $\begin{array}{l}\text { Planeación y uso } \\
\text { del tiempo }\end{array}$ & 74 & 3.37 & 0.49 & 68 & 3.17 & 0.57 & 127 & 3.63 & 0.39 & 0.000 \\
\hline & Metodología & 74 & 3.51 & 0.41 & 68 & 3.36 & 0.53 & 127 & 3.75 & 0.26 & 0.000 \\
\hline & Evaluación & 74 & 3.42 & 0.41 & 68 & 3.20 & 0.60 & 127 & 3.70 & 0.36 & 0.000 \\
\hline & \begin{tabular}{ll}
\multicolumn{3}{l}{ Relación entre el } \\
maestro y los \\
alumnos, $10 s$ \\
alumnos y los \\
maestros entre sí
\end{tabular} & 74 & 3.52 & 0.43 & 68 & 3.27 & 0.57 & 127 & 3.73 & 0.33 & 0.000 \\
\hline & $\begin{array}{l}\text { Reflexión y } \\
\text { sensibilización }\end{array}$ & 74 & 3.70 & 0.43 & 68 & 3.66 & 0.47 & 127 & 3.89 & 0.26 & 0.000 \\
\hline
\end{tabular}

Nota: Licenciatura en educación física y deporte: LEFyD, Licenciatura en enseñanza de las matemáticas: LEM y Licenciatura en educación especial: LEE.

En la tabla 3 se presentan los resultados otorgados a la GEPIA de acuerdo con el semestre que estaban cursando los estudiantes en formación docente involucrados en el estudio. A través de un análisis de ANOVA con un nivel de confianza del $95 \%$ se obtuvieron valores no significativos ( $p$-valor $>0.05$ ) en cinco de las seis categorías del cuestionario. Sin embargo la categoría de reflexión y sensibilización tuvo un p-valor altamente significativo ( $\mathrm{p}$-valor $<0.010)$ entre los semestres analizados. Para conocer exactamente qué semestres se encontraban las diferencias estadísticas se realizó la prueba post hoc de Tukey. A través de sus resultados se puede afirmar que las diferencias significativas se encuentran entre el segundo, el sexto y octavo semestre con p-valores significativos $(<0.050)$ ya que los estudiantes de los últimos semestres (sexto y octavo) otorgan puntajes más altos en la dimensión de reflexión y sensibilización.

Tabla 3. Perspectiva de prácticas inclusivas según semestre

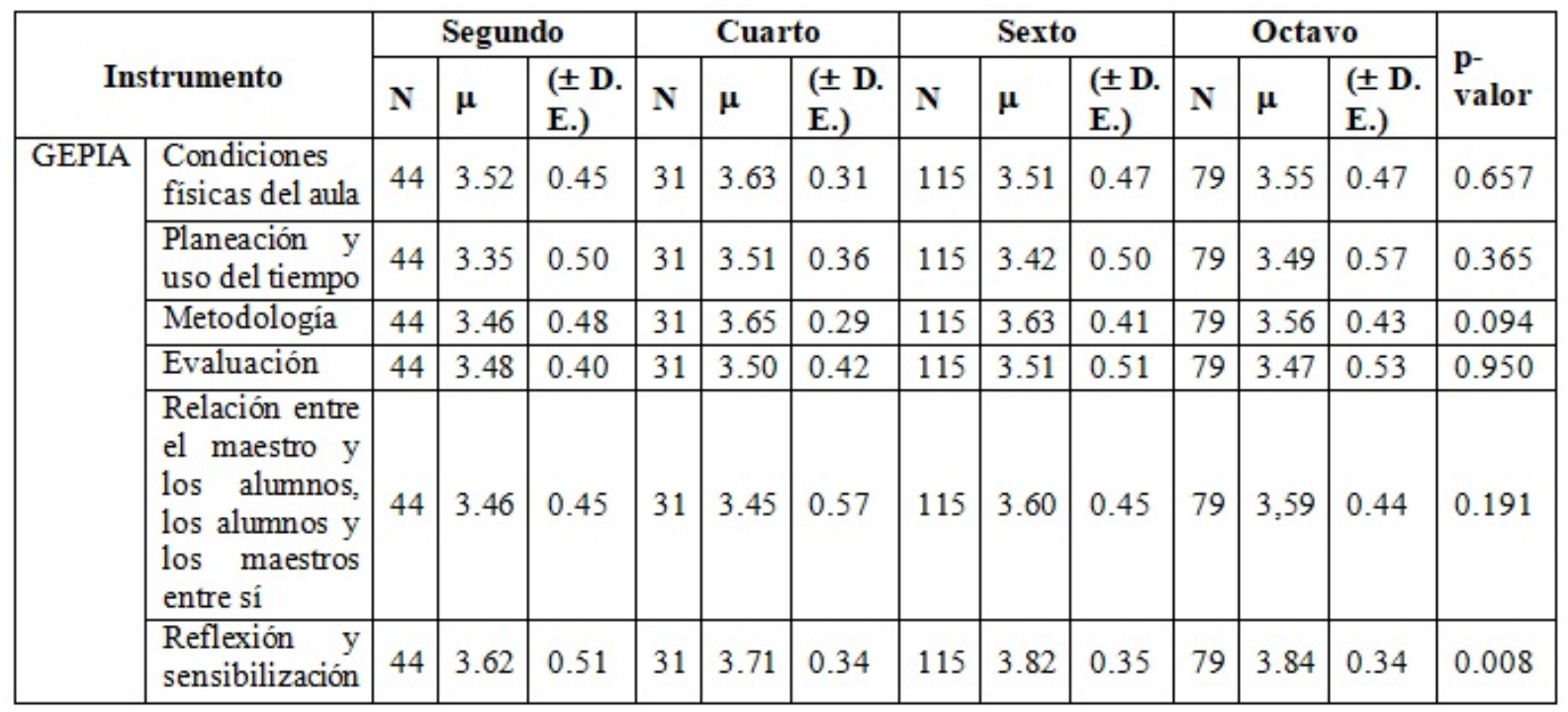




\section{DiSCUSIÓN Y CONCLUSIONES}

Partimos de la premisa que la educación inclusiva debe garantizar el acceso, permanencia, participación, aprendizaje y el egreso de todos los niños, niñas, adolescentes y jóvenes de educación regular, con especial énfasis en aquellos que están excluidos, marginados o en riesgo de estarlo. Bajo el principio de equidad debe mejorar las prácticas pedagógicas a través de un conjunto de acciones orientadas a eliminar o minimizar las barreras que limitan el aprendizaje y la participación social; mismas que surgen de la interacción entre los estudiantes y su contexto educativo; así como las políticas, instituciones, culturas y las prácticas sociales.

Con fundamento en los hallazgos de la presente investigación se puede identificar que existe una diferencia altamente significativa entre las opiniones de los estudiantes por sexo en relación a las categorías que refiere la GEPIA en la puesta de prácticas inclusivas; reflejándose que las mujeres están más sensibilizadas para atender a la diversidad a diferencia que los hombres. Estas diferencias pueden explicarse, desde nuestro punto de vista; por la influencia que se tiene sobre la formación en inclusión que paulatinamente se va abordando en la licenciatura en educación especial donde el porcentaje de mujeres frente a hombres es significativamente más alto. Por otra parte, tal como lo puntualiza Blanco (2004) en referencia al ejercicio docente de la mujer "es una mirada distinta [...] más rica, más amplia y más comprensiva, capaz de acoger más aspectos, más ámbitos y más protagonistas [...]” (p. 48). Es importante referir que tanto la licenciatura en educación física y deporte, así como la licenciatura en enseñanza de las matemáticas es visible un porcentaje mayor en hombres; sin embargo el perfil de egreso de ambas carreras no centra su atención en la construcción de prácticas inclusivas.

Por otra parte, se identifica que existen diferencias altamente significativas entre las opiniones que tienen los estudiantes de las tres licenciaturas que se ofertan; identificándose que los estudiantes de la licenciatura en educación especial están más familiarizados y sensibilizados para emprender prácticas inclusivas, siguiendo los estudiantes de educación física y deporte; y reflejándose puntajes más bajos en los estudiantes de enseñanza de las matemáticas. Este resultado tiene estrecha relación al perfil de egreso que cada carrera oferta. Una muestra de ello es que el documento curricular de la licenciatura en educación especial puntualiza

"Formar profesionales que posean las competencias [...] que le permitan desempeñarse con calidad en [...] la detección, intervención y seguimiento pedagógico para la eliminación de barreras para el aprendizaje y la participación de la población con discapacidad, trastorno, aptitudes sobresalientes y grupos en situación de riesgo o desventaja; facilitando la inclusión educativa, social y laboral con equidad, pertinencia y calidad" (Facultad de Ciencias de la Educación, 2019, p.75).

El plan de estudios de esta licenciatura centra su atención en contenidos curriculares relacionados a didácticas para atender a la diversidad de la población escolar, así como para construir y/o generar ambientes inclusivos. Por su parte, en el perfil de egreso de la licenciatura en educación física y deporte se identifica una competencia especifica centrada en "decidir y adecuar estrategias didácticas para poder incorporar personas con discapacidad o vulnerabilidad en el desarrollo de programas educativos relativos a la cultura física para el cumplimiento de la educación incluyente" (Facultad de Ciencias de la Educación, 2015a, p. 61). En el mapa curricular de esta carrera profesional se identifica una asignatura optativa relacionada a la educación física inclusiva. Finalmente, en el programa educativo de la licenciatura en enseñanza de las matemáticas sólo se identifica la asignatura optativa denominada Detección de niños con necesidades educativas especiales; misma que refleja una intervención centrada en la diversidad. Por tanto, el perfil de egreso está centrado en "formar profesionales con competencias que les permita identificar y resolver problemas relacionados a la enseñanza y aprendizaje desde un enfoque del pensamiento aritmético, algebraico, geométrico, estocástico y variacional (Facultad de Ciencias de la Educación, 2015b). Centrando la atención en estudiantes en formación docentes; lo anterior descrito; da pauta para identificar la importancia de considerar en el perfil de egreso y en las competencias especificas contenidos que reflejen la atención a la diversidad escolar; con la finalidad de promover prácticas inclusivas y generar ambientes incluyentes en las aulas y en la comunidad escolar. 
Aún con la poca o nula información que tienen los estudiantes en formación docente en torno a la educación inclusiva o a las prácticas inclusivas; buscan mecanismos para solventar su práctica pedagógica y dar cumplimiento a los compromisos en las aulas regulares. Es de gran relevancia promover una cultura inclusiva en las aulas de los futuros docentes, centrada en promover buenas prácticas inclusivas que permita atender a la diversidad que prevalece en las escuelas. Es evidente que los programas de estudio de la Facultad de Ciencias de la Educación de la Universidad de Colima, tiene un sentido incluyente en su didáctica; sin embargo los resultados de la presente investigación reflejan que falta camino por recorrer para hacer realidad que los estudiantes en formación docente ejerzan prácticas inclusivas en las aulas regulares.

El papel de toda institución formadora de docentes es formar profesionales, científicos y ciudadanos responsables, autónomos y comprometidos para actuar en beneficio de la sociedad y su entorno; por tanto, implica poner en práctica las competencias profesionales y específicas adquiridas durante su formación académica. El campo laboral requiere expertos preparados para crear, imaginar, producir, innovar y reinventarse en su ámbito de acción interactuando con equipos de diversas profesiones. Nos encontramos ante una tarea y una oportunidad docente; si partimos de la premisa respecto a que hablar de diversidad escolar implica pensar y construir la mejor escuela, que es sin lugar a dudas la escuela inclusiva; si no estamos actuando en ese aspecto, nuestros estudiantes se convierten en población vulnerable al no satisfacer sus necesidades de formación y, por tanto, de su futura profesión. Desde una visión inclusiva, las escuelas formadoras de docentes son vistas como una inspiración, un reto a vencer para poder lograr la inclusión educativa y social. Son también el escenario que apertura, pero que también se adapta y reconstruye una nueva forma de ser y comportarse frente al tema de atención a la diversidad; de cultivar prácticas inclusivas.

Finalmente es importante puntualizar que nos decidimos analizar las prácticas inclusivas de los estudiantes en formación docente porque queremos contribuir en la elaboración de propuestas para mejorar la formación que se oferta en la actualidad, por ello que la discusión sobre este tema nos ayuda a comprender cómo perciben los futuros docentes la atención a la diversidad en las aulas escolares. La Organización para la Cooperación y el Desarrollo Económico -OCDE- (2016) puntualiza la importancia que los programas de formación en licenciatura recuperen mejor las visiones de lo que implica ser profesionista, para que se enfoquen en preparar jóvenes con las herramientas básicas que les permita competir en espacios laborales y ascender a lo largo de su trayectoria. Consideramos que tenemos conocimientos insuficientes sobre el tema, por lo que es preciso y valioso saber más de las circunstancias culturales que constituyen las prácticas inclusivas de los jóvenes en formación docente. De acuerdo con la UNESCO (2014), en la actualidad el número de maestros calificados, la práctica docente y la formación universitaria afrontan graves problemas sistémicos en el mundo entero, por lo que consideramos que hablar y discutir respecto a las prácticas inclusivas es un tema pertinente para la agenda de la investigación y para las practicas educativas.

\section{REFERENCIAS}

Booth, T. y Ainscow, M. (2002). Index for inclusion. Developing learning and participation in schools. CSIE. Disponible en http://www.csie.org.uk/resources/translations/IndexEnglish.pdf

Booth, T. y Ainscow, M. (2015). Guía para la educación inclusiva. Desarrollando el aprendizaje y la participación en los centros escolares. España: Organización de Estados Iberoamericanos-FUHEM.

Blanco, G. N. (2004). El saber de las mujeres en la educación. Revista de Educación, 6(3), 43-53.

Facultad de Ciencias de la Educación, Universidad de Colima (2015a). Documento curricular de la Licenciatura en Educación Física y Deporte. México.

Facultad de Ciencias de la Educación, Universidad de Colima (2015b). Documento curricular de la Licenciatura en Enseñanza de las Matemáticas. México.

Facultad de Ciencias de la Educación, Universidad de Colima (2019). Documento curricular de la Licenciatura en Educación Especial. México. 
García, I., Romero, S., Rubio, S., Flores, V. \& Martínez, A. (2015). Comparación de Prácticas Inclusivas de Docentes de Servicios de Educación Especial en México. Actualidades investigativas, 15(3), 238-254.

García, I., Romero, S, y Escalante, L. (2011). Diseño y validación de la guía de evaluación de las prácticas inclusivas en el aula GEPIA. Documento presentado en el XI Congreso nacional de investigación educativa. México.

Jiménez, R. (2014).Educación especial. Un seminario invisible. México: Universidad Autónoma de Tlaxcala.

Lopes, S. (2015). Políticas de inclusão e a crise da educação especial. Critica Educativa, 1(1), 147-162.

Márquez, N.; Vargas, M.; Ramos, B. (2016). La dimensión internacional del curriculum para la formación del licenciado en educación especial. En J. Hernández y G. Amador. La dimensión internacional del curriculum. Los primeros pasos... en la Universidad de Colima. México. UCOL.

Organización para la Cooperación y el Desarrollo Económico -OCDE- (2016). Panorama de la Educación 2016. México. Paris: Organización para la Cooperación y el Desarrollo. Disponible en: https://www.oecd.org/educat ion/skills-beyond-school/EAG2016-Mexico.pdf Acceso en 27/06/2019

Organización de las Naciones Unidas para la Educación, la Ciencia y la Cultura-UNESCO- (2004). Temario abierto sobre educación inclusiva. Inglaterra. Disponible en: http://www.unesco.org/unesco/temario/education/ Acceso el 13/02/2019

Organización de las Naciones Unidas para la Educación, la Ciencia y la Cultura-UNESCO- (2014). Formación de docentes. Francia: Organización de las Naciones Unidas para la Educación, la Ciencia y la Cultura. Disponible en: http://www.unesco.org/new/es/unesco/themes/icts/teacher-education/ Acceso el 13/03/2019

Orjuela, G. (2009). Formación pedagógica e investigativa en la licenciatura en educación especial de la Universidad Pedagógica Nacional. Revista pedagogía y saberes, (31), 52-57.

Secretaría de Educación Pública -SEP- (2006). Propuesta de intervención educativa para alumnos y alumnas con aptitudes sobresalientes. México: SEP. Disponible en https://elisaee.files.wordpress.com/2013/03/propuesta-de -intevenciefbfbdn.pdf Acceso el 03/04/2020

Universidad de Colima (2010). Visión 2030. Universidad de Colima, Universidad sin fronteras. México. Disponible en https://portal.ucol.mx/content/docrevista/documento_1052.pdf Acceso el 03/04/2020

\section{BY-NC-SA}

